\title{
DETERMINANT LINE BUNDLE ON MODULI SPACE OF PARABOLIC BUNDLES
}

\author{
INDRANIL BISWAS
}

\begin{abstract}
In [BR1], [BR2], a parabolic determinant line bundle on a moduli space of stable parabolic bundles was constructed, along with a Hermitian structure on it. The construction of the Hermitian structure was indirect: The parabolic determinant line bundle was identified with the pullback of the determinant line bundle on a moduli space of usual vector bundles over a covering curve. The Hermitian structure on the parabolic determinant bundle was taken to be the pullback of the Quillen metric on the determinant line bundle on the moduli space of usual vector bundles. Here a direct construction of the Hermitian structure is given. For that we need to establish a version of the correspondence between the stable parabolic bundles and the Hermitian-Einstein connections in the context of conical metrics. Also, a recently obtained parabolic analog of Faltings' criterion of semistability plays a crucial role.
\end{abstract}

\section{INTRODUCTION}

Let $X$ be a compact connected Riemann surface of genus $g$ and $S \subset X$ a finite nonempty subset; if $g=0$, then $\# S \geq 3$. Fix a positive integer $r$. For each point $s \in S$, fix sequences

$$
r=r_{s, 1}>r_{s, 2}>\cdots>r_{s, \ell_{s}}>r_{s, \ell_{s}+1}=0 .
$$

and $0 \leq \alpha_{1}(s)<\alpha_{2}(s)<\cdots<\alpha_{\ell_{s}}(s)<1$. Let $\mathcal{M}_{P}$ be the moduli space of sable parabolic vector bundles of rank $r$ and degree $d$ over $X$ associated to this data.

In [BR1], BR2, generalizing the determinant line bundle over the moduli space of usual vector bundles, a parabolic determinant line bundle was constructed which is a holomorphic Hermitian line bundle over $\mathcal{M}_{P}$. Fix a ramified Galois covering

$$
f: Y \longrightarrow X
$$

such that $\mathcal{M}_{P}$ is identified with a moduli space $\mathcal{M}_{Y}(\Gamma)$ of stable $\Gamma$-linearized vector bundles, where $\Gamma=\operatorname{Gal}(f)$, over $Y$.

It was shown that the parabolic determinant line bundle is holomorphically identified with the usual determinant line bundle over $\mathcal{M}_{Y}(\Gamma)$. The Hermitian structure on the parabolic determinant line bundle was obtained by pulling back the Quillen metric on the usual determinant line bundle over $\mathcal{M}_{Y}(\Gamma)$. Our aim here is to give a direct construction of the Hermitian structure on the parabolic determinant line bundle.

2000 Mathematics Subject Classification. 14F05, 14D20.

Key words and phrases. Parabolic bundle, determinant bundle, Quillen metric. 
Fix a conical metric $h$ on $X$. We show that any stable parabolic vector bundle $E_{*} \in$ $\mathcal{M}_{P}$ admits a unique Hermitian-Einstein connection with respect to $h$ (see Theorem 3.2). This Hermitian-Einstein structure plays a crucial role in the construction of the Hermitian structure on the parabolic determinant line bundle.

Faltings' semistability criterion says that a vector bundle $E$ on a smooth complex projective curve $C$ is semistable if and only if there is a vector bundle $F \longrightarrow C$ such that $H^{i}(C, E \otimes F)=0$ for $i=0,1[\mathrm{Fa}]$. This criterion has the following generalization for parabolic vector bundles.

Fix $X$ and parabolic divisor $S$ as above. Fix a positive integer $N$; all the parabolic weights will be assumed to be integral multiples of $1 / N$. There is a parabolic vector bundle $V_{*}^{0}$ with the following property: a parabolic vector bundle $E_{*}$ is parabolic semistable if and only if there is another parabolic vector bundle $F_{*}$ such that

$$
H^{i}\left(X,\left(E_{*} \otimes F_{*} \otimes V_{*}^{0}\right)_{0}\right)=0
$$

for $i=0,1$, where $\left(E_{*} \otimes F_{*} \otimes V_{*}^{0}\right)_{0}$ is the vector bundle underlying the parabolic tensor product $E_{*} \otimes F_{*} \otimes V_{*}^{0}$. (See [Bi2].)

The above parabolic vector bundle $V_{*}^{0}$ is the other key input in the construction of the Hermitian structure on the parabolic determinant line bundle.

\section{PRELIMinaRies}

Let $X$ be a compact connected Riemann surface. Fix a finite subset

$$
\emptyset \neq S \subset X
$$

A parabolic vector bundle over $X$ with $S$ as the parabolic divisor is a vector bundle $E$ on $X$ equipped with a parabolic structure over $S$, meaning for each point $s \in S$, we have

- a filtration

$$
E_{s}=E_{s, 1} \supset E_{s, 2} \supset \cdots \supset E_{s, \ell_{s}} \supset E_{s, \ell_{s}+1}=0 .
$$

of subspaces of the fiber $E_{s}$, and

- rational numbers

$$
0 \leq \alpha_{1}(s)<\alpha_{2}(s)<\cdots<\alpha_{\ell_{s}}(s)<1 .
$$

The sequence in (2.2) is called the quasiparabolic filtration, and the sequence in (2.3) is called the parabolic weights. The points of $S$ are called parabolic points. For notational convenience, a vector bundle $E$ with a parabolic structure will be denoted by $E_{*}$.

The parabolic degree of $E_{*}$ is defined to be

$$
\operatorname{par}-\operatorname{deg}\left(E_{*}\right):=\operatorname{degree}(E)+\sum_{s \in S} \sum_{i=1}^{\ell_{s}} \alpha_{\ell_{s}}(s) \cdot \operatorname{dim} E_{s, i} / E_{s, i+1} .
$$

Any subbundle $F$ of $E$ has an induced parabolic structure. We recall that a parabolic vector bundle $E_{*}$ is called semistable if for all proper subbundles $F \subset E$ of positive rank, 
the inequality

$$
\frac{\operatorname{par}-\operatorname{deg}\left(F_{*}\right)}{\operatorname{rank}\left(F_{*}\right)} \leq \frac{\operatorname{par}-\operatorname{deg}\left(E_{*}\right)}{\operatorname{rank}\left(E_{*}\right)}
$$

holds, where $F_{*}$ is the parabolic vector bundle defined by the induced parabolic structure on $F$. If the strict inequality

$$
\frac{\operatorname{par}-\operatorname{deg}\left(F_{*}\right)}{\operatorname{rank}\left(F_{*}\right)}<\frac{\operatorname{par}-\operatorname{deg}\left(E_{*}\right)}{\operatorname{rank}\left(E_{*}\right)}
$$

holds then $E_{*}$ is called stable.

A semistable parabolic vector bundle is called polystable if it is a direct sum of stable parabolic vector bundles.

Fix a positive integer $r$, and also fix an integer $d$. Let $\mathcal{M}_{P}$ denote the moduli space of stable parabolic vector bundles of rank $r$ and degree $d$ with a fixed type of quasiparabolic filtration and fixed parabolic weights. (See [MS] for the construction of $\mathcal{M}_{P}$ as well as some of its properties.)

We will assume that $S$ and the parabolic data are such that $\operatorname{dim} \mathcal{M}_{P}>0$. Note that this assumption rules out the case where $X=\mathbb{C P}^{1}$ and $\# S=1$. Indeed, if $X=\mathbb{C P}^{1}$ with $\# S=\{s\}$, then using the Grothendieck's theorem that any vector bundle over $\mathbb{C P}^{1}$ splits into a direct sum of line bundles it follows that any semistable parabolic vector bundle $E_{*}$ must be of the following form:

$$
E=L^{\oplus r}
$$

where $L \longrightarrow \mathbb{C P}^{1}$ is a line bundle, and the quasiparabolic filtration is the trivial filtration

$$
0 \subset E_{s} .
$$

A theorem due to Faltings says that a vector bundle $E$ over $X$ is semistable if and only if there is another vector bundle $E^{\prime}$ such that $E \otimes E^{\prime}$ is cohomologically trivial, meaning

$$
H^{0}\left(X, E \otimes E^{\prime}\right)=0=H^{1}\left(X, E \otimes E^{\prime}\right)
$$

Fa]. This criterion can be generalized to the context of parabolic vector bundles in the following way. Fix a positive integer $N$. Consider parabolic vector bundles with parabolic weights integral multiples of $1 / N$ (with arbitrary quasiparabolic structure and rank).

Lemma 2.1 ([Bi2] $)$. Then there is a parabolic vector bundle $V_{*}^{0}$ with the following property: A parabolic vector bundle $E_{*}$ is semistable if and only if there is a parabolic vector bundle $E_{*}^{\prime}$ such that the vector bundle $\left(E_{*} \otimes E_{*}^{\prime} \otimes V_{*}^{0}\right)_{0}$ underlying the parabolic tensor product $E_{*} \otimes E_{*}^{\prime} \otimes V_{*}^{0}$ is cohomologically trivial.

It should be emphasized that such a parabolic vector bundle $V_{*}^{0}$ can be explicitly constructed. We recall a construction of a parabolic vector bundle $V_{*}^{0}$ that satisfies the above condition.

Fix a Galois covering

$$
f: Y \longrightarrow X
$$


such that

- $f$ is ramified exactly over $S$, and

- the ramification index of each point in $f^{-1}(S)$ is $N-1$, where $N$ is the fixed integer such that all the parabolic weights are integral multiples of $1 / N$.

See [Na, p. 26, Proposition 1.2.12] for the existence of $f$ satisfying these conditions. Let

$$
\Gamma:=\operatorname{Gal}(f)
$$

be the Galois group for the covering $f$. There is a natural bijective correspondence between the following two classes:

(1) all $\Gamma$-linearized vector bundles on $Y$, and

(2) the parabolic vector bundles over $X$ for which the parabolic divisor in contained in $S$ and all the parabolic weights are integral multiples of $1 / N$.

(See [Bi1].)

Consider the trivial vector bundle over $Y$

$$
\widetilde{V}:=\mathcal{O}_{Y} \otimes_{\mathbb{C}} \mathbb{C}(\Gamma),
$$

where $\mathbb{C}(\Gamma)$ is the group algebra of $\Gamma$ defined in (2.5). The action of $\Gamma$ on $Y$ produces an action of $\Gamma$ on $\mathcal{O}_{Y}$. The natural action of $\Gamma$ on $\mathbb{C}(\Gamma)$ and the action of $\Gamma$ on $\mathcal{O}_{Y}$ together define a $\Gamma$-linearization on the vector bundle $\widetilde{V}$ in (2.6) . Let

$$
V_{*}^{0} \longrightarrow X
$$

be the parabolic vector bundle over $X$ corresponding to the $\Gamma$-linearized vector bundle $\widetilde{V}$. This parabolic vector bundle $V_{*}^{0}$ satisfies the condition in Lemma 2.1, (See [Bi2], [BH].)

\section{Conical metric on $X$}

Fix a positive integer $N$.

Let $S$ be the subset in (2.1). Set

$$
X^{\prime}:=X \backslash S
$$

Let $\mathbb{D}:=\{z \in \mathbb{C}|| z \mid<1\}$ be the unit disk. For a point $x \in X$, a holomorphic coordinate around $x$ is a holomorphic embedding

$$
\varphi: \mathbb{D} \longrightarrow X
$$

such that $\varphi(x)=0$.

A conical metric on $X$ of order $N$ is a Hermitian metric $h$ on the holomorphic tangent bundle $T X^{\prime}$ satisfying the following condition: For each point $x \in D$, there is a holomorphic coordinate around $x$

$$
\varphi: \mathbb{D} \longrightarrow X
$$


such that

$$
\left.h\right|_{\mathbb{D}}=f(z) \frac{d z \otimes d \bar{z}}{|z|^{2(N-1) / N}},
$$

where $f: \mathbb{D} \longrightarrow \mathbb{R}^{+}$is a smooth function; $\varphi(\mathbb{D})$ is identified with $\mathbb{D}$ using $\varphi$.

Let $E_{*}$ be a parabolic vector bundle over $X$. Let $E$ be the vector bundle underlying the parabolic bundle $E_{*}$.

A Hermitian structure on $E_{*}$ is a Hermitian structure $H$ on $\left.E\right|_{X^{\prime}}$ satisfying the following condition: Take any point $s \in S$, and take any holomorphic section $\sigma$ of $E$ defined around $s$. If $\sigma(s)$ is a nonzero element of $E_{s, i} \subset E_{s}$ (see (2.2)), then

$$
\|s\|_{H}=f(z)|z|^{\alpha_{i}(s)}
$$

(see (2.3) for $\alpha_{i}(s)$ ), where $z$ is a holomorphic coordinate around $s$, and $f$ is a smooth function with values in positive real numbers.

Fix a conical metric of order $N$ on $X$. Let $\omega$ be the corresponding Kähler form on $X^{\prime}$.

Let $E_{*}$ be a parabolic vector bundle over $X$ with the property that all the parabolic weights of $E_{*}$ are integral multiples of $1 / N$.

Definition 3.1. A Hermitian structure $H$ on $E_{*}$ is called Hermitian-Einstein if the curvature of the Chern connection on $\left.E\right|_{X^{\prime}}$ for $H$ is of the form

$$
\lambda \cdot \operatorname{Id}_{E} \otimes \omega,
$$

where $\lambda$ is some constant positive real number.

Theorem 3.2. Let $E_{*}$ be a stable parabolic vector bundle over $X$ such that all the parabolic weights of $E_{*}$ are integral multiples of $1 / N$. Then $E_{*}$ admits a Hermitian-Einstein structure. If $H$ and $H_{1}$ are two Hermitian-Einstein structures on $E_{*}$, then

$$
H_{1}=c \cdot H,
$$

where $c$ is a constant real positive number.

Proof. Let $f: Y \longrightarrow X$ be the covering in (2.4). The pullback of the (fixed) conical metric on $X$ defines a Hermitian structure on $f^{-1}(X \backslash S)$. From the definition of the conical metric it follows that this Hermitian metric on $f^{-1}(X \backslash S)$ extends to a Hermitian metric on $Y$. Let $\widetilde{\omega}$ be the Kähler form on $Y$ associated to this Hermitian structure on $Y$.

Let

$$
W \longrightarrow Y
$$

be the $\Gamma$-linearized vector bundle corresponding to the parabolic vector bundle $E_{*}$. Since $E_{*}$ is parabolic stable, it follows that the corresponding $\Gamma$-linearized vector bundle $W$ is polystable [BBN, p. 349, Proposition 4.1]. Consequently, $W$ admits a Hermitian-Einstein structure [Do]. The Hermitian-Einstein structure is not unique, but the HermitianEinstein connection is unique. From the uniqueness of the Hermitian-Einstein connection 
it follows immediately that the action of $\Gamma$ on $W$ preserves the Hermitian-Einstein connection. Consequently, for any Hermitian-Einstein structure $H_{W}$ on $W$, the Hermitian form

$$
H_{W}^{\prime}:=\sum_{\gamma \in \Gamma} \gamma^{*} H_{W}
$$

is Hermitian-Einstein. Clearly, the action of $\Gamma$ on $W$ preserves the Hermitian form $H_{W}^{\prime}$. Therefore, $H_{W}^{\prime}$ descends to a Hermitian structure on $\left.E\right|_{X \backslash S}$. It is now straight-forward to check that this Hermitian structure on $\left.E\right|_{X \backslash S}$ is a Hermitian structure on the parabolic vector bundle $E_{*}$.

From the definition of a Hermitian-Einstein structure on $E_{*}$ it follows that any two Hermitian-Einstein forms on $E_{*}$ differ by an automorphism of the parabolic vector bundle $E_{*}$. Since $E_{*}$ is parabolic stable, all parabolic automorphisms are constant scalar multiplications. Hence any two Hermitian-Einstein forms on $E_{*}$ differ by multiplication with a constant real number. This completes the proof of the theorem.

\section{The Determinant Line BUNDLE}

Let $\mathcal{E} \longrightarrow X \times T$ be a holomorphic vector bundle, where $T$ is a complex manifold. We will consider $\mathcal{E}$ as a holomorphic family of vector bundles over $X$ parametrized by $T$. Let

$$
p: X \times T \longrightarrow T
$$

be the projection. The direct images $R^{0} p_{*} \mathcal{E}$ and $R^{1} p_{*} \mathcal{E}$ are coherent analytic sheaves on $T$. Therefore,

$$
\operatorname{det}\left(R^{i} p_{*} \mathcal{E}\right):=\bigwedge^{\text {top }} R^{i} p_{*} \mathcal{E}
$$

$i=0,1$, are holomorphic line bundles over $T$ (see $[\mathrm{Ko}, \mathrm{Ch} . \mathrm{V}, \S 6]$ for the construction of the determinant line bundle of a coherent analytic sheaf). If $T$ is algebraic, and $\mathcal{E}$ is an algebraic vector bundle, then the line bundle $\operatorname{det}\left(R^{i} p_{*} \mathcal{E}\right)$ is also algebraic.

The determinant of the family $\mathcal{E}$ is defined to be the line bundle

$$
d(\mathcal{E}):=\operatorname{det}\left(R^{0} p_{*} \mathcal{E}\right)^{*} \otimes \operatorname{det}\left(R^{1} p_{*} \mathcal{E}\right) \longrightarrow T .
$$

Let

$$
\mathcal{E}_{*} \longrightarrow X \times T
$$

be a family of parabolic vector bundles of fixed quasiparabolic type and fixed parabolic weights. Let

$$
p_{X}: X \times T \longrightarrow X
$$

be the natural projection. Consider the parabolic vector bundle $V_{*}^{0}$ constructed in (2.7). So $p_{X}^{*} V_{*}^{0}$ is a constant family of parabolic vector bundles parametrized by $T$. Let

$$
\mathcal{E}_{*} \otimes p_{X}^{*} V_{*}^{0} \longrightarrow X \times T
$$


be the family of parabolic vector bundles obtained by taking the parabolic tensor product. Let

$$
\left(\mathcal{E}_{*} \otimes p^{*} V_{*}^{0}\right)_{0} \longrightarrow X \times T
$$

be the family of vector bundles underlying the family of parabolic vector bundles in (4.5).

Definition 4.1. The parabolic determinant bundle for the family $\mathcal{E}_{*}$ is defined to be the line bundle

$$
d\left(\left(\mathcal{E}_{*} \otimes p^{*} V_{*}^{0}\right)_{0}\right) \longrightarrow T
$$

(see (4.2)). The parabolic determinant bundle for $\mathcal{E}_{*}$ will be denoted by $p d\left(\mathcal{E}_{*}\right)$.

Let $\mathcal{M}_{P}$ be a moduli space of stable parabolic vector bundles over $X$ of rank $r$ and degree $d$ with a fixed type of quasiparabolic filtration and fixed parabolic weights. In general, there is no universal parabolic vector bundle over $X \times \mathcal{M}_{P}$. However, every point $z \in \mathcal{M}_{P}$ has an open neighborhood $U_{z}$ in étale topology such that there is a universal parabolic vector bundle over $X \times U_{z}$. Hence there is an open neighborhood $U_{z}^{\prime}$ of $z$ in analytic topology such that there is a universal parabolic vector bundle over $X \times U_{z}^{\prime}$.

Using the locally defined universal parabolic vector bundles on $\mathcal{M}_{P}$, we can construct locally defined parabolic determinant bundles.

Fix a point $x_{0} \in X$. Let $\mathcal{E}_{*}$ be a locally defined (in either étale or analytic topology) universal parabolic vector bundles over $X \times U$. The vector bundle over $U$ obtained by restricting the underlying vector bundler $\mathcal{E}$ to $\left\{x_{0}\right\} \times U$ will be denoted by $\mathcal{E}_{x_{0}}$.

Assume that $T$ is connected. Hence the function $\mathcal{M}_{P} \longrightarrow \mathbb{Z}$ that sends any $E_{*} \in \mathcal{M}_{P}$ to

$$
\chi:=\operatorname{dim} H^{0}(X, E)-\operatorname{dim} H^{1}(X, E)
$$

is a constant one.

Lemma 4.2. The locally defined line bundles

$$
\operatorname{pd}\left(\mathcal{E}_{*}\right)^{\otimes r} \otimes\left(\bigwedge^{r} \mathcal{E}_{x_{0}}\right)^{\otimes \chi}
$$

(see Definition 4.1) patch together naturally to define an algebraic line bundle over $\mathcal{M}_{P}$.

Proof. Let $E_{*}$ be a stable parabolic vector bundle. Take any automorphism $\tau$ of the underlying vector bundle $E$ that preserves the quasiparabolic filtrations. Since $E_{*}$ is stable, we know that $\tau=\lambda \cdot \operatorname{Id}_{E}$ for some $\lambda \in \mathbb{C}^{*}$. Using this it follows that if $\mathcal{E}_{*}$ and $\mathcal{E}_{*}^{\prime}$ are two universal parabolic vector bundles over $X \times U$, then there is a natural line bundle $L \longrightarrow U$ and a canonical isomorphism

$$
\mathcal{E}_{*}^{\prime}=\mathcal{E}_{*} \otimes f^{*} L
$$

where $\phi: X \times U \longrightarrow U$ is the projection. In fact, we may take

$$
L:=\phi_{*}\left(\operatorname{Hom}\left(\mathcal{E}_{*}, \mathcal{E}_{*}^{\prime}\right)\right)
$$


(here Hom is the sheaf of parabolic homomorphisms). In this case, the isomorphism in (4.7) is given by the natural pairing

$$
\operatorname{Hom}\left(\mathcal{E}_{*}, \mathcal{E}_{*}^{\prime}\right) \otimes \mathcal{E}_{*} \longrightarrow \mathcal{E}_{*}^{\prime} .
$$

From (4.7) and the projection formula it follows that

$$
\operatorname{pd}\left(\mathcal{E}_{*}\right)=\operatorname{pd}\left(\mathcal{E}_{*}^{\prime}\right) \otimes L^{\otimes \chi},
$$

where $\chi$ is defined in (4.6). On the other hand, from (4.7),

$$
\bigwedge^{r} \mathcal{E}_{x_{0}}^{\prime}=\left(\bigwedge^{r} \mathcal{E}_{x_{0}}\right) \otimes L^{\otimes r}
$$

From (4.8) and (4.9),

$$
\operatorname{pd}\left(\mathcal{E}_{*}\right)^{\otimes r} \otimes\left(\bigwedge^{r} \bigwedge^{r} \mathcal{E}_{x_{0}}\right)^{\otimes \chi}=p d\left(\mathcal{E}_{*}^{\prime}\right)^{\otimes r} \otimes\left(\bigwedge^{r} \bigwedge^{r} \mathcal{E}_{x_{0}}^{\prime}\right)^{\otimes \chi}
$$

This completes the proof of the lemma.

Definition 4.3. Let

$$
\mathcal{D} \longrightarrow \mathcal{M}_{P}
$$

be the holomorphic line bundle obtained from Lemma 4.2.

There is a parabolic determinant line bundle on $\mathcal{M}_{P}$; see [BR1] and [BR2] for its construction.

Lemma 4.4. The holomorphic line bundle $\mathcal{D}$ in Definition 4.3 coincides with the parabolic determinant line bundle.

Proof. Take any parabolic vector bundle $E_{*} \in \mathcal{M}_{P}$. Let

$$
W \longrightarrow Y
$$

be the $\Gamma$-linearized vector bundle corresponding to $E_{*}$, where $Y$ is the Galois covering in (2.4). For $i=0,1$, we have

$$
H^{i}\left(X,\left(\mathcal{E}_{*} \otimes p^{*} V_{*}^{0}\right)_{0}\right)=H^{i}(Y, W)
$$

$[\mathrm{BH}$, p. 252, (15)] (in $[\mathrm{BH}$, p. 252, (15)] it is proved for $i=0$, but the proof is identical for $i=1$; see [Bi2, p. 327, (8)]). Using (4.10) it is straight-forward to check that $\mathcal{D}$ coincides with the parabolic determinant line bundle.

\section{Hermitian structures}

5.1. Hermitian structure on $V^{0}$. Let $V^{0}$ denote the vector bundle underlying the parabolic bundle $V_{*}^{0}$ in (2.7). We will first construct a Hermitian structure on the restriction of $V^{0}$ to

$$
X^{\prime}:=X \backslash S \subset X
$$


Consider the group $\Gamma$ in $(2.5)$. Let $h_{\Gamma}$ be the inner product on the group algebra $\mathbb{C}(\Gamma)$ defined by

$$
h_{\Gamma}\left(\sum_{z \in \Gamma} c_{z} \cdot z, \sum_{z \in \Gamma} d_{z} \cdot z\right)=\sum_{z \in \Gamma} c_{z} \overline{d_{z}} .
$$

This inner product $h_{\Gamma}$ defines a Hermitian structure on the trivial vector bundle $\widetilde{V}=$ $\mathcal{O}_{Y} \bigotimes_{\mathbb{C}} \mathbb{C}(\Gamma)$ in (2.6) $)$. The action of the Galois group $\Gamma=\operatorname{Gal}(f)$ on $\tilde{V}$ preserves this Hermitian structure. The pullback $f^{*}\left(\left.V^{0}\right|_{X^{\prime}}\right)$ is identified with the restriction of $\widetilde{V}$ to $f^{-1}\left(X^{\prime}\right) \subset Y$, where $X^{\prime}$ is the open subset in (5.1). Consequently, there is a unique Hermitian structure on $\left.V^{0}\right|_{X^{\prime}}$ such that the identification of $f^{*}\left(\left.V^{0}\right|_{X^{\prime}}\right)$ with $\left.\widetilde{V}\right|_{f^{-1}\left(X^{\prime}\right)}$ is an isometry.

Definition 5.1. Let $h_{0}$ denote the Hermitian structure on $\left.V^{0}\right|_{X^{\prime}}$ constructed above.

It is straight-forward to check that $h_{0}$ is a Hermitian structure on the parabolic vector bundle $V_{*}^{0}$.

The parabolic vector bundle $V_{*}^{0}$ is polystable. Hence it has a Hermitian-Einstein structure (see Theorem 3.2). The following proposition is obtained by comparing the constructions of the Hermitian structure $h_{0}$ (see Definition 5.1) and Hermitian-Einstein structure in Theorem 3.2.

Proposition 5.2. The Hermitian structure $h_{0}$ on the polystable parabolic vector bundle $V_{*}^{0}$ is a Hermitian-Einstein form.

5.2. Hermitian structure on $\mathcal{D}$. Fix a positive integer $N$ such that all the parabolic weights in the parabolic data associated to $\mathcal{M}_{P}$ are integral multiples of $1 / N$. Fix a conical metric $h$ of order $N$ on $X$.

Let $E_{*} \in \mathcal{M}_{P}$ be a stable parabolic vector bundle. Let $H$ be a Hermitian-Einstein form on $E_{*}$. From Theorem 3.2 we know that $H$ is determined uniquely up to a constant scalar.

The Hermitian form $h_{0}$ on $\left.V^{0}\right|_{X^{\prime}}$ (see (5.1)) and the Hermitian-Einstein form $H$ on $E_{*}$ together define a Hermitian-Einstein structure on $\left.\left(E \otimes V^{0}\right)\right|_{X^{\prime}}$, where $X^{\prime}$ is defined in (5.1). This Hermitian structure on $\left.\left(E \otimes V^{0}\right)\right|_{X^{\prime}}$ defines a Hermitian structure on the parabolic tensor product $E_{*} \otimes V_{*}^{0}$. Let $\widehat{H}$ denote the Hermitian structure on the parabolic vector bundle $E_{*} \otimes V_{*}^{0}$ given by the above Hermitian structure on $\left.\left(E \otimes V^{0}\right)\right|_{X^{\prime}}$.

Let $\left(E_{*} \otimes V_{*}^{0}\right)_{0}$ be the vector bundle underlying the parabolic vector bundle $E_{*} \otimes V_{*}^{0}$.

The Hermitian structure $\widehat{H}$ and the conical metric $h$ together define a Hermitian structure on the vector space $H^{0}\left(X,\left(E_{*} \otimes V_{*}^{0}\right)_{0}\right)$.

The Hermitian structure $\widehat{H}$ induces a Hermitian structure on

$$
C^{\infty}\left(X ;\left(E_{*} \otimes V_{*}^{0}\right)_{0} \otimes \Omega_{X}^{0,1}\right) .
$$

The restriction of it to the orthogonal complement of the image of the Dolbeault operator for the holomorphic vector bundle $\left(E_{*} \otimes V_{*}^{0}\right)_{0}$

$$
\bar{\partial}_{\left(E_{*} \otimes V_{*}^{0}\right)_{0}}: C^{\infty}\left(X ;\left(E_{*} \otimes V_{*}^{0}\right)_{0}\right) \longrightarrow C^{\infty}\left(X ;\left(E_{*} \otimes V_{*}^{0}\right)_{0} \otimes \Omega_{X}^{0,1}\right)
$$


defines a Hermitian structure on the vector space $H^{1}\left(X,\left(E_{*} \otimes V_{*}^{0}\right)_{0}\right)$.

Let

$$
\Delta_{\left(E_{*} \otimes V_{*}^{0}\right)_{0}}:=\left(\bar{\partial}_{\left(E_{*} \otimes V_{*}^{0}\right)_{0}}\right)^{*} \bar{\partial}_{\left(E_{*} \otimes V_{*}^{0}\right)_{0}}
$$

be the Laplacian of the operator in (5.2).

Consider the complex line

$$
p d\left(E_{*}\right):=\bigwedge^{\text {top }} H^{0}\left(X,\left(E_{*} \otimes V_{*}^{0}\right)_{0}\right)^{*} \otimes \bigwedge^{\text {top }} H^{1}\left(X,\left(E_{*} \otimes V_{*}^{0}\right)_{0}\right)
$$

(compare it with Definition 4.1). The above inner products on $H^{0}\left(X,\left(E_{*} \otimes V_{*}^{0}\right)_{0}\right)$ and $H^{1}\left(X,\left(E_{*} \otimes V_{*}^{0}\right)_{0}\right)$ together produce an inner product on $p d\left(E_{*}\right)$. Using the Quillen's construction, we modify this inner product on $\operatorname{pd}\left(E_{*}\right)$ using the eigenvalues of the Laplacian $\Delta_{\left(E_{*} \otimes V_{*}^{0}\right)_{0}}$ defined in (5.3) (see $[\mathrm{Qu}]$ ). This procedure produces a Hermitian structure on the holomorphic line bundle $p d\left(\mathcal{E}_{*}\right) \longrightarrow T$ (see Definition 4.1 ). Hence we get a Hermitian structure on the holomorphic line bundle

$$
\mathcal{D} \longrightarrow \mathcal{M}_{P}
$$

constructed in Definition 4.3 .

Definition 5.3. The above Hermitian structure on the holomorphic line bundle $\mathcal{D} \longrightarrow$ $\mathcal{M}_{P}$ will be denoted by $H_{p Q}$.

Consider the covering $f$ in (2.4). As noted in the proof of Theorem 3.2, the pullback of the conical metric $h$ by $f$ produces a Hermitian metric on $Y$. As before, the Kähler form on $Y$ associated to this Hermitian metric will be denoted by $\widetilde{\omega}$.

Let

$$
W \longrightarrow Y
$$

be the $\Gamma$-linearized vector bundle corresponding to $E_{*}$, where $Y$ is the Galois covering in (2.4). Let

$$
\bar{\partial}_{W}: C^{\infty}(Y ; W) \longrightarrow C^{\infty}\left(Y ; W \otimes \Omega_{Y}^{0,1}\right)
$$

be the Dolbeault operator for the holomorphic vector bundle $W$. Since the vector bundle $W$ is polystable, it has a Hermitian-Einstein structure. Using this Hermitian-Einstein structure and the Kähler form $\widetilde{\omega}$ on $Y$ we define the Laplacian

$$
\Delta_{W}:=\bar{\partial}_{W}^{*} \bar{\partial}_{W}
$$

It can be shown that the eigenvalues of $\Delta_{W}$, along with their multiplicities, coincide with those of the operator $\Delta_{\left(E_{*} \otimes V_{*}^{0}\right)_{0}}$ constructed in (5.3). Indeed,

$$
C^{\infty}(Y ; W) \quad \text { and } \quad C^{\infty}\left(Y ; W \otimes \Omega_{Y}^{0,1}\right)
$$

are identified with $C^{\infty}\left(X ;\left(E_{*} \otimes V_{*}^{0}\right)_{0} \otimes \Omega_{X}^{0,1}\right)$ and $C^{\infty}\left(X ;\left(E_{*} \otimes V_{*}^{0}\right)_{0}\right)$ respectively, and these identifications preserve the inner products. Furthermore, these identifications take the differential operator $\bar{\partial}_{\left(E_{*} \otimes V_{*}^{0}\right)_{0}}$ to $\bar{\partial}_{W}$. Consequently, these identification takes $\Delta_{\left(E_{*} \otimes V_{*}^{0}\right)_{0}}$ to $\Delta_{W}$. Hence the eigenvalues of $\Delta_{W}$, along with their multiplicities, coincide with those of the operator $\Delta_{\left(E_{*} \otimes V_{*}^{0}\right)_{0}}$. 
Therefore, we have the following proposition:

Proposition 5.4. The holomorphic isomorphism in Lemma 4.4 between $\mathcal{D}$ and the parabolic determinant line bundle takes in Hermitian structure $H_{p Q}$ in Definition 5.3 to the Hermitian structure on the parabolic determinant line bundle.

See [BR1], [BR2] for the construction of the Hermitian structure on the parabolic determinant line bundle.

\section{REFERENCES}

[BBN] V. Balaji, I. Biswas and D. S. Nagaraj: Principal bundles over projective manifolds with parabolic structure over a divisor, Tohoku Math. Jour. 53 (2001), 337-367.

[BR1] I. Biswas and N. Raghavendra: Determinants of parabolic bundles on Riemann surfaces, Proc. Indian Acad. Sci. (Math. Sci.) 103 (1993), 41-71.

[BR2] I. Biswas and N. Raghavendra: Curvature of the determinant bundle and the Kähler form over the moduli of parabolic bundles for a family of pointed curves, Asian Jour. Math. 2 (1998), 303-324.

[Bi1] I. Biswas: Parabolic bundles as orbifold bundles, Duke Math. Jour. 88 (1997), 305-325.

[Bi2] I. Biswas: A cohomological criterion for semistable parabolic vector bundles on a curve, Comp. Ren. Acad. Sci. Math. 345 (2007), 325-328.

[BH] I. Biswas and G. Hein: Parabolic Raynaud bundles, Manuscr. Math. 126 (2008), 247-253.

[Do] S. K. Donaldson: A new proof of a theorem of Narasimhan and Seshadri, Jour. Diff. Geom. 18 (1983), 269-277.

[Fa] G. Faltings: Stable G-bundles and projective connections, Jour. Alg. Geom. 2 (1993), 507-568.

[Ko] S. Kobayashi: Differential geometry of complex vector bundles, Princeton University Press, Princeton, NJ, Iwanami Shoten, Tokyo, 1987.

[MS] V. Mehta and C. S. Seshadri: Moduli of vector bundles on curves with parabolic structures, Math. Ann. 248 (1980), 205-239.

[Na] M. Namba: Branched coverings and algebraic functions, Pitman Research Notes in Mathematical Series, no. 161, Longman Scientific and Technical, Harlow; John Wiely \& Sons, New York, 1987.

[Qu] D. G. Quillen: Determinants of Cauchy-Riemann operators over a Riemann surface, Funct. Anal. Appl. 19 (1985), 31-34.

School of Mathematics, Tata Institute of Fundamental Research, Homi Bhabha Road, BOMBAY 400005, INDIA

E-mail address: indranil@math.tifr.res.in 